\title{
Patient perspectives on transitioning to amyotrophic lateral sclerosis multidisciplinary clinics
}

This article was published in the following Dove Press journal: Journal of Multidisciplinary Healthcare

\author{
Kerri Lynn Schellenberg' \\ Gregory Hansen ${ }^{2}$ \\ 'Department of Medicine, Division \\ of Neurology, University of \\ Saskatchewan, Saskatoon, SK, Canada; \\ ${ }^{2}$ Department of Pediatrics, Division \\ of Critical Care, University of \\ Saskatchewan, Saskatoon, SK, Canada
}

Correspondence: Kerri Lynn Schellenberg

Department of Medicine, Division of Neurology, University of Saskatchewan, 3-616 10th Street East, Saskatoon, SK S7H 0G9, Canada

$\mathrm{Tel}+\mid 30693$ | 2858

Fax + I 306 93। 2847

Email kerri.schellenberg@usask.ca
Purpose: Multidisciplinary clinics (MDC) have become the standard of care for management of amyotrophic lateral sclerosis (ALS). No studies however, have captured patients' perspectives during a transition to ALS MDCs. Recently, an ALS MDC emerged from a single-physician clinic in Saskatoon, Canada, providing patients with a unique exposure to two different models of care.

Patients and methods: Fifteen patients with ALS participated in semi-structured interviews that were digitally recorded and transcribed. Two independent researchers performed an inductive thematic analysis. Information was coded based on emerging and a priori themes. An iterative process followed involving discussion and reexamination of the themes until consensus was reached.

Results: All patients cited the convenience of integrated care as an advantage. Other advantages included clinical expertise and advocacy potential. Travel and reduced mobility were the most commonly discussed barriers/disadvantages of MDC attendance. The impact of geography and weather appeared to augment both the appreciation of an integrated approach and the impediment of travel, compared to the existing literature. The need for individualized care was demonstrated by the conflicting viewpoints obtained from participants. Most patients felt additional practitioners and supports for both patients and caregivers were required.

Conclusion: ALS patients transitioned to MDCs reported many of the advantages and disadvantages reported elsewhere. A novel perspective of a MDC's advocacy potential was recognized, and the need for an innovative approach to meet demands for individualized care was highlighted. Keywords: amyotrophic lateral sclerosis, motor neuron disease, multidisciplinary care, qualitative research

\section{Introduction}

Multidisciplinary clinics (MDC) have become the standard of care for management of amyotrophic lateral sclerosis (ALS) worldwide. They have been shown to confer the following advantages in several studies: improved quality of life, lengthened survival, fewer hospital admissions with shorter duration of stays, and increased use of adaptive equipment, medication (ie, Riluzole), noninvasive ventilation, and feeding tubes. ${ }^{1-6}$ Guidelines have been created by American and European organizations which state that patients with ALS should have access to regular specialized multidisciplinary care. ${ }^{7,8}$ The Canadian ALS Best Practice Guidelines are currently in development, and will outline the importance of MDC accessibility for all Canadians with ALS. ${ }^{9}$

At present, the viewpoint of patients and caregivers regarding MDC in ALS has been limited. Through use of online surveys, Stephens et al ${ }^{10}$ explored benefits, 
disadvantages, and barriers to MDC use from patients' perspectives in the United States. Although many benefits were noted (integrated care, clinical expertise, and access to research), tiring clinic visits and long travel times were also identified. In a study from the United Kingdom, patients and caregivers stated benefits of a single point of access, expertise, convenience of integrated care, and regular followup. Patients also valued the team approach as well as the individual practitioners. ${ }^{11}$

We have previously published hospitalization data from patients in Saskatoon, Canada, from an era when a provincial ALS MDC did not exist. ${ }^{12}$ Our study suggested the need for early diagnosis and coordinated outpatient multidisciplinary management to reduce lengthy and costly hospital admissions. During the transition to an ALS MDC in Saskatoon, Canada, we solicited patient perspectives in order to optimize its development.

\section{Patients and methods Subjects}

Fifteen patients who attended the University of Saskatchewan's MDC in Saskatoon were recruited to participate in the interview process. Patients were eligible for participation if they were over 18 years of age and diagnosed with ALS. The exclusion criteria included cognitive impairment. Patients with speech impairment were encouraged to participate if they could communicate using adaptive devices or through their caregivers if present during the interview. An information sheet was provided to every patient who attended the ALS clinic until recruitment was complete. This information sheet explained the study in a standardized fashion in accordance with our institution's Ethics Board. Written consent was obtained for each patient. Demographic and disease stage information was not obtained in order to protect participant anonymity. The University of Saskatchewan granted ethics approval.

\section{Local context}

Saskatchewan is a Canadian province with a population of over 1.1 million people, and a geographical area of 651,036 $\mathrm{km}^{2}$. The newly formed MDC for ALS is located in Saskatoon, Saskatchewan, and is affiliated with the University of Saskatchewan. At the time of data collection, a neurologist, physiatrist, respirologist, clinic coordinator, speech language pathologist, physical therapist, respiratory therapist, and part-time nursing support serviced the growing clinic. ALS care had been previously provided through a single-physician clinic model.

\section{Methods}

Patients with ALS were telephone interviewed and audio recorded by a trained contractor from the University of Saskatchewan's Social Sciences Research Laboratories. The semi-structured interviewing process ensured that standardized information was collected, but allowed for individual opinions to be expressed.

The interview template was based on a previously administered local quality assurance survey and an article by Stephens et al $^{10}$ entitled "A Qualitative Study of Multidisciplinary ALS Clinic Use in the United States". Together five common queries emerged for our interview: 1) advantages seen in attending an MDC, 2) disadvantages of attending an MDC, 3) barriers that might prevent attendance, 4) the role of travel distance and MDC attendance, and 5) recommendations for the current MDC model.

After the interviews were transcribed, an anonymized version was provided to the two researchers and evaluated according to inductive thematic analysis. ${ }^{13}$ Fifteen themes were coded and organized into the five categories above. Discrepancies were resolved through an iterative process of discussion and reexamination of the themes until consensus was reached.

\section{Results}

Fifteen patients with ALS participated in the interviews between May and October of 2017. Caregivers of five patients were present during the phone call and clarified the comments provided by participants; caregivers were asked to facilitate telephone communication in those with significant dysarthria. The interviews ranged from 8 to 44 minutes, and average interview time was 16 minutes. Fifteen codes were identified, and all codes were categorizable according to the five common queries as outlined by our interview questions. The categories and codes were as follows: 1) advantages - clinical expertise, communication among experts, convenience of integrated care, information, research, advocacy; 2) disadvantages - travel/mobility, scheduling, parking, clinic visit rigidity, lengthy appointments, duplication of services; 3) barriers - travel (duplicate code); 4) impact of travel on MDC attendance - benefits outweigh difficulties of travel; 5) recommendations to current MDC model - human resources, telehealth technology.

\section{Advantages of a MDC}

All patients believed that the convenience of receiving integrated care from multiple practitioners in one setting was a definite advantage in the MDC model of care. 
“... really helpful to just go to one place and to be seen by multiple people instead of having to go to all different places at different times. We've had to do that, it's harder trying to do that."

"Well, I think the biggest advantage is the fact that we get to see so many doctors without having to run around and trying to make appointments... It's a lot of information to take in in the short term... but it's definitely a benefit. It's going to be even more of a benefit as things progress and mobility gets worse, you know, with it all being right there and being to see many people. That's fantastic."

The vast majority $(n=12,87 \%)$ cited clinical expertise as a strong advantage of the MDC.

“... you go to see different people [in the community] and they've never dealt with anybody with ALS before and you know they're not quite sure how to handle things and trying to get a hold of doctors is all really difficult. And it ends up being quite a lengthy process."

"Well, I find that I can get some answers. I went almost 2 years and nobody... seemed to know anything. Just keep bouncing from one to the other... so you go to a [multidisciplinary clinic], they can check your respiratory [function], they can check your symptoms... find out what's going on."

Patients $(n=2 ; 13 \%)$ also identified communication among experts as a benefit since multiple practitioners in one clinic can result in joint decision-making and improved communication.

"... the client's history would be passed from one to the other right there and if there's any questions they'd be talking to each other."

"... each one coming in one at a time and they're check-

ing you out...very organized."

Some patients felt that obtaining information about the disease was a positive aspect of MDC attendance $(n=6$; $40 \%$ ), but others were ambivalent, or even averse to hearing additional information.

"It's something I don't really want to think too much about it because it's an ugly thing to think about."

"I don't go on the computer and spend hours looking about it, in fact I don't do it at all."

"I just don't want to know."

Access to research was important to the majority of clinic attendees interviewed $(n=8 ; 53 \%)$. "...hopefully everybody would be up to date on what the current research and literature is and what opportunities are there for studies. And hopefully because there is an actual ALS clinic you would be able to participate in studies there as well."

"We just think that it has the potential to ensure better services for ALS patients in the end."

Multiple interviewees commented on the advocacy potential of the burgeoning clinic at a health region and governmental level $(\mathrm{n}=5 ; 33 \%)$.

"Okay, and you just lobby 'em Saskatchewan government people and get after them."

"Well I just hope it keeps going. Like I said, I think this is a very good thing."

\section{Disadvantages of a MDC}

Although 53\% $(n=15)$ of patients stated that they could not think of any disadvantages of attending an MDC, difficulties pertaining to transportation were addressed by almost all participants $(n=13 ; 87 \%)$. Travel distance and reduced mobility were the main factors listed as impediments to MDC attendance.

"It's just so hard for people in rural Saskatchewan... I'm 3 hours from Saskatoon, there's people that live a lot further too... But at least we have somewhere to go now."

"I'm in a wheelchair, and all I can move is my hand... it's so hard to get to appointments now...."

Lengthy appointments were also cited as a disadvantage $(n=2 ; 13 \%)$; however, it was noted that this was a necessary consequence of seeing multiple practitioners in one setting.

"ALS is a disease, as you know, that is exhausting for patients.

So, to come for a long period of time is exhausting for patients. But you know, it's kind of a double-edged sword, right? Either you're coming back and forth a lot, or you're coming for a longer period of time and getting exhausted from it. So, it's just kind of the nature of the beast."

"It's so convenient to be able to make one appointment - and granted, the appointment takes a couple, 3 hours... but you get to see so many different people."

Duplication of services was seen as a disadvantage for the minority $(n=2 ; 13 \%)$.

"When we went [to clinic] we saw a physiotherapist... we already do have one that comes out to our home when we need her." 


\section{Barriers to MDC attendance and impact of travel}

Travel impediments were the only barrier to clinic attendance cited by participants ( $\mathrm{n}=13 ; 87 \%)$. Particularly, inclement winter weather was volunteered as a major hurdle by the large minority $(\mathrm{n}=7 ; 47 \%)$.

"It takes a lot of energy for someone with ALS to get... in your wheelchair or just get from point A to point B. You just don't jump in the car and take off... it's quite a procedure doing it every time we go... And this is Saskatchewan remember. We have 40 below [Celsius]."

“....in Saskatchewan you never know what the weather's gonna be like in winter."

Several patients commented that the benefit of MDC attendance outweighed the identified travel barrier in the following statements:

"But you just gotta make exceptions and go a day or two early if the weather looks funny. Because it's something I don't believe you want to miss... there's lots of benefits to it, so I want to make sure that we can be there. No, if it was a single appointment and we had to go five different times that would make it a whole lot harder than doing it this way."

"I would worry more about the clinic being good than having to travel... even if you have to travel a bit further, it would be worth it."

\section{Recommendations for additions to the current MDC model}

Two-thirds of patients had recommendations for additional services to enhance care in the Saskatoon MDC. Two themes emerged: the need for further human resources and the need for emotional support for both patient and caregiver. The care practitioners requested by two or more participants included dietitians, dental hygienists, and supportive services such as social work and spiritual care. One patient admitted to extreme confusion regarding the roles of the clinic practitioners and services available in the community, and $33 \%(n=5)$ believed additional help should be available to navigate the disease process. The request for additional supports to help patients and families was compelling.

"I wouldn't mind seeing something more for the caregiver... my caregiver is my wife, you know, she's kind of lost..."

Although travel and mobility were barriers to clinic attendance, the participants had divergent opinions about the use of telehealth and other technologies.
"I think the ability even just to do some Skype appointments would be good. I know I've done a telehealth before and did not like it at all because I actually had to go to the university to make the appointment, whereas I could have just stayed home and done it over Skype."

"I don't really care for Skype or anything like that. I'd rather sit down and have the conversation with the doctor or the person that's helping me."

\section{Discussion}

While our qualitative study demonstrated many advantages and disadvantages of ALS MDCs consistent with those previously seen in the literature, we report several noteworthy findings. These include the following: 1) patient viewpoints obtained during the transition to a MDC; 2) the need for an individualized approach; and 3) the advocacy potential of a MDC.

The transition to an ALS MDC provided us with a novel opportunity to collect data with the aim of building a patientcentered clinic. Our participants were uniquely positioned to compare their previous experience of receiving care to a MDC approach. The fact that all patients cited advantages of the MDC approach is further weight in favor of ALS MDC, which is the recognized standard of care. The European Federation of Neurologic Sciences have published guidelines which outline the recommended components and processes of an ALS MDC. ${ }^{8}$ It is notable that the gaps in service providers that our patients identified (dietitians, social work, spiritual care, and dental care providers) are the very same listed as necessary components of the ALS MDC in the European Federation of Neurologic Sciences guidelines. This demonstrates concordance between patient perspectives and the existing guidelines. The aim of this study was to incorporate these perspectives to create a patient-centered clinic.

The conflicting statements from participants regarding several issues explored by the interviews demonstrate the need for an individualized approach to patient care. Although some patients believe that obtaining information about ALS is an advantage of the MDC, others are averse to receiving an overload of information or state they simply do not wish to know details. The same is true of research; only half of the patients felt that access to research was important. While some patients welcomed the idea of telemedicine, several patients preferred to sit down with their care providers face to face. Stephens et $\mathrm{al}^{10}$ discussed the importance of individualizing the MDC experience since patients stated clinic visits could be lengthy and exhausting, a sentiment that was also evident in our study. These findings demonstrate the 
importance of flexibility in patient-centered care and pertain not only to the content of the clinic visit discussions, but also to the types of providers relevant to a specific patient/ presentation, and with respect to the location of the visit (in person vs telemedicine).

Although advocacy is a recognized by-product of neuromuscular MDC, ${ }^{14}$ this is the first study to demonstrate that patients view the advocacy potential of a MDC ALS clinic as a benefit. This could be due to the fact that our MDC is a fledgling clinic, and patients recognize the importance of its development. Two-thirds of our patients called for additional services and supports, and the need for additional personnel and emotional support for both patients and caregivers was a theme, which emerged from the interviews. This study alludes to the importance of the clinic to aid with patient and family navigation of the disease process, provide emotional support, reduce duplication of services, promote research opportunities, and maximize patient access and participation in clinic. In this study, patients viewed the clinic as positioned to approach government/health region to advocate on their behalf.

Our Canadian perspective on ALS MDCs highlights the impact of geography and weather on clinic attendance. The convenience of multiple appointments in one setting might be particularly germane in Saskatchewan; access to care can prove challenging in this Canadian landscape where vast geographical areas with sparse populations are served by a distant tertiary care center. Patients unanimously perceived integrated care at a single appointment as advantageous, which is higher than previously reported (Stephens et $\mathrm{al}^{10}$ $33 \%$; O'Brien et $\mathrm{al}^{11}$ did not report percentages). Similarly, the advantage of clinical expertise was also more commonly cited in our study ( $87 \%$ ) compared with the study by Stephens et $\mathrm{al}^{10}(32 \%)$. This might also reflect geography and resource allocation, as clinical expertise may be more valued with relatively fewer specialists and/or tertiary centers available. Travel distance and reduced mobility were seen as the main disadvantages or impediments to clinic attendance (87\%) and more commonly addressed than reported by Stephens et $\mathrm{al}^{10}(15 \%)$. Again, we suspect that the potentially long distances patients traverse to our clinic may be responsible for this discrepancy. Given the provincial weather patterns with winter blizzards and extreme cold warnings, almost half (47\%) of our patients volunteered that inclement weather was a barrier to clinic attendance. As such, it seems particularly important that alternatives such as satellite clinics, telehealth, and home videoconferencing should be explored for those who cannot travel or abide lengthy visits. This observation could be generalizable to urban populations of greater density, since clinic access remains an issue, regardless of address.

Limitations of this study include a relatively small sample size $(n=15)$, but this is consistent with other qualitative interview studies in ALS that have been recently published, each with 16 participants. ${ }^{15,16}$ The generalizability of our findings may be limited, since many national and international MDC are already well established. However, patients from larger urban centers may lack the comparative experience of a single-physician clinic.

\section{Conclusion}

In this Canadian study of patients' perspectives regarding ALS MDC, the impact of geography and weather on clinic attendance appeared to augment both the appreciation of an integrated approach, and the impediment of travel. Further study may be required to add to the small body of literature regarding the use of telemedicine and alternatives in ALS care to mitigate this barrier. To our knowledge, this is the first study soliciting patients' opinions during the development of an MDC ALS clinic and provided a novel perspective from patients who had previous experiences from a single-physician clinic. An innovative approach to MDC is required to meet the demands for an individualized approach, additional supports, and to realize the advocacy potential of a MDC.

\section{Acknowledgement}

The University of Saskatchewan's Department of Medicine research grant funded the study. No honorarium or other form of payment was provided to any author to produce the manuscript.

\section{Disclosure}

The authors report no other conflicts of interest in this work.

\section{References}

1. Rooney J, Byrne S, Heverin M, et al. A multidisciplinary clinic approach improves survival in ALS: a comparative study of ALS in Ireland and Northern Ireland. J Neurol Neurosurg Psychiatry. 2015;86(5):496-501.

2. Traynor BJ, Alexander M, Corr B, Frost E, Hardiman O. Effect of a multidisciplinary amyotrophic lateral sclerosis (ALS) clinic on ALS survival: a population based study, 1996-2000. J Neurol Neurosurg Psychiatry. 2003;74(9):1258-1261.

3. Aridegbe T, Kandler R, Walters SJ, Walsh T, Shaw PJ, Mcdermott CJ. The natural history of motor neuron disease: assessing the impact of specialist care. Amyotroph Lateral Scler Frontotemporal Degener. 2013;14(1):13-19.

4. van den Berg JP, Kalmijn S, Lindeman E, et al. Multidisciplinary ALS care improves quality of life in patients with ALS. Neurology 2005;65(8):1264-1267. 
5. Chiò A, Bottacchi E, Buffa C, Mutani R, Mora G, and the PARALS. Positive effects of tertiary centres for amyotrophic lateral sclerosis on outcome and use of hospital facilities. J Neurol Neurosurg Psychiatry. 2006;77(8):948-950.

6. Cordesse V, Sidorok F, Schimmel P, Holstein J, Meininger V. Coordinated care affects hospitalization and prognosis in amyotrophic lateral sclerosis: a cohort study. BMC Health Serv Res. 2015;15:134.

7. Miller RG, Jackson CE, Kasarskis EJ, et al. Practice Parameter update: the care of the patient with amyotrophic lateral sclerosis: multidisciplinary care, symptom management, and cognitive/behavioral impairment (an evidence-based review. Neurology. 2009;73:1227-1233.

8. FNS Task Force on Diagnosis and Management of Amyotrophic Lateral Sclerosis, Andersen PM, Abrahams S, et al. EFNS guidelines on the clinical management of amyotrophic lateral sclerosis (MALS)--revised report of an EFNS task force. Eur J Neurol. 2012;19(3):360-375.

9. Theme 14 Multidisciplinary care and improving quality of life. Amyotroph Lateral Scler Frontotemporal Degener. 2017;18(sup2):305-324.

10. Stephens HE, Young J, Felgoise SH, Simmons Z. A Qualitative Study of Multidisciplinary ALS Clinic Use in the United States. Amyotrophic Lateral Sclerosis and Frontotemporal Degeneration. 2016;17(1-2):55-61.
11. O’Brien M, Jack B, Douglas Mitchell J, Whitehead JB, Mitchell JD. Multidisciplinary team working in motor neurone disease: patient and family carer views. British Journal of Neuroscience Nursing. 2011;7(4):580-585.

12. Gunton A, Hansen G, Schellenberg KL. Hospital utilization for patients with amyotrophic lateral sclerosis in saskatoon, Canada. Amyotroph Lateral Scler Frontotemporal Degener. 2018;19(3-4):1-5.

13. Braun V, Clarke V. Using thematic analysis in psychology. Qual Res Psychol. 2006;3(2):77-101.

14. Paganoni S, Nicholson K, Leigh F, et al. Developing multidisciplinary clinics for neuromuscular care and research: Multidisciplinary Care for Neuromuscular Disease. Muscle Nerve. 2017;56:848-858.

15. Aoun SM, Connors SL, Priddis L, Breen LJ, Colyer S. Motor Neurone Disease family carers' experiences of caring, palliative care and bereavement: an exploratory qualitative study. Palliat Med. 2012;26(6): $842-850$.

16. Baxter SK, Baird WO, Thompson S, et al. The impact on the family carer of motor neurone disease and intervention with noninvasive ventilation. J Palliat Med. 2013;16(12):1602-1609.
Journal of Multidisciplinary Healthcare

\section{Publish your work in this journal}

The Journal of Multidisciplinary Healthcare is an international, peerreviewed open-access journal that aims to represent and publish research in healthcare areas delivered by practitioners of different disciplines. This includes studies and reviews conducted by multidisciplinary teams as well as research which evaluates the results or conduct of such teams or health
Dovepress

care processes in general. The journal covers a very wide range of areas and welcomes submissions from practitioners at all levels, from all over the world. The manuscript management system is completely online and includes a very quick and fair peer-review system. Visit http://www.dovepress.com/ testimonials.php to read real quotes from published authors.

Submit your manuscript here: https://www.dovepress.com/journal-of-multidisciplinary-healthcare-journal 